\title{
Historical Evolution and Development: Formation of Multi-ethnic Complementary Patterns in Yunnan Tibetan Area
}

\author{
Xuekun Li \\ College of Economics and Management \\ Yunnan Agricultural University \\ Kunming, China 650201
}

\author{
Yuqin Zhang* \\ College of Economics and Management \\ Yunnan Agricultural University \\ Kunming, China 650201 \\ *Corresponding Author
}

\begin{abstract}
The Yunnan Tibetan Area refers to the Diqing Tibetan Autonomous Prefecture of Yunnan Province. It is the Yunnan Tibetan Area in "Tibet and the Tibetan Areas of the Four Provinces (Qinghai, Sichuan, Yunnan, and Gansu)" mentioned in the Sixth Tibet Work Symposium. During the long-term history of evolution, dated from the earliest multiethnic activities, the history of Yunnan Tibetan Area can be mainly divided into following four stages: the conflict between Tang, Tubo and Nanzhao; Chieftain Mu's rule; the great unification period of the Yuan Dynasty; and Gushi Khan of the Heshuote tribe of Mongolia defeated Chieftain Mu. Against the background of conflict and collision, the multi-ethnic complementary advancement and the construction of the identification of the same region have gradually taken shape. Though different in ethnic groups, the multi-ethnic symbiosis pattern of the Tibetan areas in the same region and the governance system of the integration of politics and religion has been consolidated in the long-term development of history.
\end{abstract}

Keywords-Yunnan Tibetan area; complementary symbiosis; different in ethnic groups; identification of the same region

\section{INTRODUCTION}

The Yunnan Tibetan area refers to the Diqing Tibetan Autonomous Prefecture of Yunnan Province. It is the Yunnan Tibetan area in "Tibet and the Tibetan Areas of the Four Provinces (Qinghai, Sichuan, Yunnan, and Gansu)" mentioned in the Sixth Tibet Work Symposium. There are 26 ethnic groups in Yunnan Tibetan Area: Tibetan nationality, Li-Su nationality, Han nationality, Yi nationality, Bai nationality, Miao nationality, Hui nationality, Naxi nationality, Pumi nationality, etc., with a total population of 405,000. Tibetans account for $35.8 \%$ [1], thus becoming an ethnic group with most population in this area. In the long process of development, the multi-ethnic relationship in Yunnan Tibetan area has experienced a complicated and tortuous process. Compared with other Tibetan areas, the history of Yunnan Tibetan area has its own distinct characteristics. Since the Neolithic Period, there have been the traces of human beings in the Tibetan areas of Yunnan. From the 7th century AD, a large number of Tibetans entered the Yunnan Tibetan area from the Tibetan Plateau, and performed "integration, confrontation and integration" with local aboriginal ancestors, which is a historical scroll of the evolution of long-term ethnic relations. The historical pattern of multi-ethnic embedded distribution in Tibetan areas of Yunnan gradually formed.

The Yunnan Tibetan area is geographically far from the Tibetan cultural center and away from the Han cultural center, making it culturally retain the characteristics of Tibetan areas on the Tibet Plateau on the one hand. On the other hand, it has long been an important "national corridor" for movement and migration of Qiang, Di, Rong and other ethnic groups in the history, and the meeting point of the Tibet, Han, Naxi, Bai and other ethnic groups, thus forming a phenomenon of coexistence of various ethnic relations that distinguish itself from general Tibetan areas. [2]

The historical evolution of the multi-ethnic relationship in Yunnan Province is not only in line with the main evolutionary characteristics of all minorities in China, but also carries the special historical connotation of multi-ethnic relationship in Yunnan Tibetan area. Against the background of the openness, development and information globalization full of changes, the harmonious and stable multi-ethnic relationship in Yunnan Tibetan area is also facing new issues of social governance method and social changes. The multiethnic relationship in Yunnan Tibetan area will surely encounter situations and risks more complex and unpredictable than ever before. Therefore, the study on multi-ethnic relationship in Yunnan Tibetan area requires attention on the dynamic changes and reality of history and nationality in this region.

\section{THE ORIGIN OF THE MULTI-ETHNIC COMPLEMENTARY PATTERN IN YUNNAN TIBETAN AREA}

\section{A. The Earliest Multi-ethnic Activities in the Tibetan Areas of Yunnan}

According to the archaeological results of the recently unearthed cultural relics, it has been confirmed that there are traces of Tibetan ancestors living in Yunnan Province in the late Paleolithic period. The "Gordon Neolithic Culture" site, 
which has been around 4,000 years old, proves this conclusion. In particular, the "Sarcophagus Culture" site, which is about 3,000 years away from now, clearly proves that Yunnan Tibetan Area is one of the ancient residences of Tibetans and other indigenous peoples. [3] According to archaeological excavations, the Sarcophagus tombs group was discovered in Nagu, Yongzhi, Shidi of Deqin County, and Nixi Township of Zhongdian County, and bronzes, pottery, silverware, etc. It has proved that since the Neolithic Age, there have been many cultural connections between the Tibetans in Yarlung Zangbo River basin on the Tibet Plateau and in the Qamdo area in Tibet, and the Naxi, Bai, and Li-Su ethnic groups in northwest Yunnan. [4] It can be concluded that before the Qin Dynasty, there were cultural relics in the Tibetan area of Yunnan, which coexist with the Qamdo kano site culture in the upper reaches of the Lantsang River and the Cliff stone carving with more than 1,200 years of history in the Danma Mountain rock in the Renda Township of Shiya xiangdui area. [5] A cultural belt thus was formed, which fully proves that more than 2,000 years ago, the Yunnan Tibetan area has become a fusion point for economic, social, cultural exchanges and multi-ethnic integration of ethnic minorities in ancient China. From the Spring and Autumn Period to the early Han Dynasty, the traces of Tibetan ancestors living on the banks of Jinshating River and Lantsang River were distributed in Nagu of Deqin in the northwest, Yongzhi, Shidi in the southwest, and Nixi of Zhongdian in the east. [6] There are archaeological evidences that in the second and third centuries BC, the earliest Tibetan ancestors migrated from the Tibet Plateau to the Tibetan areas of Yunnan. In the first century BC, the historic sites, cemeteries and colorful cultural relics left by them were discovered in Yongzhi of Deqin, Shidi. Yunnan Tibetan community gradually entered into the Bronze Age. During this period, a variety of ethnic activities were emerged in northwestern Yunnan, including Yunnan Tibetan area.

\section{B. The Early Stage of Multi-ethnic Complementary Integration in Tibetan Areas of Yunnan}

In the first half of the 7th century, Tubo competed with the Tang Dynasty for the Erhai Sea region in the northwest of Yunnan. At the same time, in order to exert political pressure on the Nanzhao Kingdom, Tubo expelled a large number of Tibetan ancestors of the Tibet Plateau and forced them to migrate to the present Deqin, Zhongdian areas. These Tibetan ancestors gradually merged with the indigenous ancestors of Yunnan Tibetan area, becoming the origin of multi-ethnic complementary integration between Tibetan ancestors and other ethnic groups in Tibetan areas of Yunnan.

The early Tibetan ancestor who migrated southward to the Tibetan areas of Yunnan while retaining their traditional production methods, were influenced by the agricultural production techniques of other local ethnic groups. At the same time, in order to adapt to the natural environment of Yunnan Tibetan area, they cleared grasshoppers on the slopes, cut trees, planted barley, began original agricultural production, and gradually merged with local people, while developing animal husbandry and hunting industry at the bank of the Lantsang River with an altitude of 3,000 meters above sea level. The long-term co-production and coexistence of ethnic groups leads to coexistence of different ethnic groups with Tibetan nationality as the main ethnic group while many ethnic groups including minority nationalities (referring to the ancestors of Naxi, Li-Su, Yi, and Bai) and Hanshang (referring to the Han nationality). According to "A New Book of Tang's History: Nanzhao Biography", "History of Yunnan", "History of Yuan Dynasty: Geography" and other books, as early as the Tang and Song Dynasties, the two sides of Tacheng's Qizonggu Bridge have been home to Guzong, Xibo (Pumi), Moxie, Li-Su, Hanshang, Lei and other ethnic groups. So far, such phenomenon that several minority nationalities inhabited together with Iron Bridge Shenchuan River as center and complex ethnic relations have been continuously spreading to the surrounding radiation, and this feature is very prominent.

Through the understanding and judgment of the ethnic relations during this period, it can be inferred that the initial embodiment of the multi-ethnic complementary integration distribution pattern in Yunnan Tibetan area is the two-way or multi-directional flow inside the southwestern frontier. That is to say, the traditional Tibetan ancestors gradually radiate from the northeastern part with Tibet Plateau as center to the northwestern part of Yunnan Province. In addition, the ethnic groups in Yunnan Tibetan area also spreading to other areas in a centripetal manner. These flows are the basic embodiment of the inter-embedded pattern in the early Tibetan areas of Yunnan.

Compared with the "survival rationality" type of movement in the traditional society, there is a big difference between the two. The cross-regional flow of ethnic groups is more similar to the result of a "passive choice" whose purpose is mainly to satisfy its survival need. It's the ultimate result of the passive choice of different nations. It can be seen from these that the Tibetan areas in Yunnan have promoted the construction of identification of the same region among various ethnic groups during cross-regional migration in meeting their expectations of interests, eliminating confusion about the future, and enhancing ability to withstand future risks. This is not only the key to the indepth advancement of multi-ethnic inter-integration, but also the key to the multi-ethnic integration of Tibetan areas in Yunnan.

\section{Multi-ethnic Mutual AdVAnCEMENT AND CONSTRUCTION OF IDENTIFICATION OF THE SAME REGION IN TIBETAN AREAS OF YUNNAN}

It can be obviously seen from the development history of multi-ethnic complementary integration in Yunnan Tibetan area in different periods, and against various conflicts between different political powers, the harmonious coexistence of multi ethnic group results from the internal mechanism of multi-ethnic complementary integration. In terms of multi-ethnic mutual advancement and construction of identification of the same region in Tibetan areas of Yunnan, influenced by farming civilization and nomadic 
culture, the two are interrelated and interdependent in the peaceful period, forming a progressive active integration; while in the wartime period, the fight for Tibetan areas of Yunnan is performed based on the interest of different ruling classes, thus forming a passive integration. The formation of multi-ethnic complementary integration in Yunnan Tibetan area includes the active integration of multi-ethnic groups in Yunnan, but more commonly is passive integration of vulnerable minority people for survival incurred by political confrontation.

\section{A. The Period of Conflict Between Tang, Tubo and Nanzhao}

By 589 AD, the Sui Dynasty realized great unification, and later, a stronger Tang Dynasty was established. At the same time, a unified Tubo local government had been established in Tibet. With the continuous development of Nanzhao, the Yunnan Tibetan area have become an important area that Tang, Tubo and Nanzhao all competing for. Since then, the disputes among the various ethnic groups have gradually become inevitable.

In the 7th century, Tubo rose up in the Tibet Plateau. It not only fought fiercely with the Tang Dynasty in the Erhe river area of western Yunnan, but also competed with the Tang Dynasty for Helong and the Western Regions. In addition, its army also occupied Yanyuan area, Sichuan and Erhai sea area in Yunnan, seriously threatening the southwestern border of the Tang Dynasty. Facing the constant attack of Tubo, the Tang Dynasty adopted corresponding countermeasures to resolve the powerful offensive of Tubo and competed with Tubo for this strategic location in Erhai Sea region. On the one hand, the Tang Dynasty carried out fierce battle with Tubo in the Erhe region. On the other hand, the Tang Dynasty further support the Erhe area and establish close ties with Wuman tribe, Mengshezhao, and establish a ruling regime that will surrender to the Tang Dynasty to unify the tribes in Erhai. This will not only form a barrier against the expansion of Tubo's power in the southwest region, but also enable the Tang Dynasty to effectively rule the southwestern region of Yunnan and promote the growth of the Tang Dynasty. Nanzhao's founding relied mainly on the support of the Tang Dynasty, but its continuous expansion of power in order to dominate Yunnan incur a huge conflict with the interests of the Tang Dynasty, making their contradictions gradually increase and intensify, which led to the outbreak of the Tianbao War that break down the relationship between Nanzhao and the Tang Dynasty. Then, Nanzhao ran to Tubo. During the period when Nanzhao subordinated to Tubo, Tubo imposed a series of oppressive policies and cruel ethnic exploitation on Nanxun, which made Nanzhao in dire straits and the people were miserable. Therefore, Nanzhao had the idea of getting rid of Tubo and returning to the Tang Dynasty. With the constant struggle of the Tang Dynasty, Nanzhao eventually returned to the Tang Dynasty. It can be seen that during this period, the relations between ethnic groups in northwestern Yunnan were relatively complicated and chaotic. Among them, the Tibetans relying on the Tubo regime, the Han Dynasty, and Bai and Naxi peoples governed by Nanzhao are also inevitably caught in disputes because of the military struggles of the three parties. There exist suspicion and distrust among nations.

Of course, the disputes between political powers have also slowly affected the distribution of the nation. Among the various ethnic groups, Tibetans have more experience in producing and living on the plateau than other ethnic groups. Therefore, today, the occupants of Zhongdian, Deqin and northwest Weixi in the north of the Jinsha River in the Tibetan area of Yunnan are mainly Tibetans, while that of southeast is mainly Li-Su and Naxi. After the 10th century, the distribution pattern of ethnic groups in Yunnan Tibetan area has been formed. At the same time, thanks to the balanced effect of the three forces in the northwestern part of Yunnan, Yunnan has a relatively peaceful period. Since the 6th and 7th centuries, the Tibetan community in Yunnan has developed slowly and under the influence of the feudal economy and culture, it made gradual transition to feudal society.

\section{B. Chieftain Mu's Rule}

Since the 14th century, the Tibetan community in Yunnan has experienced more than 200 years of war. The predecessor of $\mathrm{Mu}$ Zeng, such as $\mathrm{Mu}$ Gao, although they won victory after the battle, built the revetment, but was soon removed by the Tibetan resistance. With such circulation, the entire Tibetan area of Yunnan has been in war. In the 17th century, feudal chieftain in Lijiang represented by Mu Zeng succeeded the Lijiang chieftain mansion, the conquest of Yunnan Tibetan area and Batang, Litang and Yanjing of the Kangba area was completed, which brought a relatively stable period to the Tibetan area of Yunnan.

In the Ming Dynasty, with the prosperity of Chieftain Mu of Naxi in Lijiang, they fought with Tibetans in the Tibetan areas of Yunnan for hundreds of times. They fought more than ten times in Jiantang Town. After the military conquest of Lijiang Chieftain, Chieftain Mu established a manor in the Tibetan area of Yunnan. The relatives of Mu's family managed Tibetans and other ethnic groups. "The managers enslaved anyone of other ethnic groups they captured". The "Shuka" system (An inherent grassroots organizational system that develops from the ancient village system and forms a specific social organization structure and function based on the region.) in Yunnan Tibetan area was replaced by "Jiang Ben" — "Mugua" — "Baisui" management system. Baisui is served by both Moxie people and the heads of the local Tibetan tribes. Nothing has changed in relevant ruling system and trade system, as they followed the original one in Tibetan areas. In terms of faith, Mu's performance is particularly prominent. They not only respect the local Tibetan Buddhism, but also pay more attention to Tibetan customary beliefs. During the two centuries of ruling Yunnan's Tibetan areas, the Mu family often recruited and used Tibetan Buddhism as a means of management in Tibetan areas of Yunnan to ease the hostility between Tibetans and Naxi people.

During the heyday of Chieftain $\mathrm{Mu}$, a new change in the ethnic relationship in Yunnan Tibetan area was the 
immigration of Naxi, Li-Su, Bai, Han and other ethnic groups, especially the large number of Naxi people. They ventured into the various areas of Yunnan and Tibet, leading to strong collision between the Naxi and the Tibetans, and promoting the unprecedented economic development and exchange of culture between Naxi and Tibet indirectly. It promoted the development of animal husbandry, agriculture, sideline and handicraft industry, and also created conditions for the development of commerce. The development of commerce has also prompted the emergence of a special group: landlords. They facilitated the exchange of merchants and promoted the development of commerce. The merchants have also been specially protected by the Mu family, and the commercial and cultural exchanges of various ethnic groups have become more convenient, further promoting the continuous development of the economy and culture of the Tibetan areas in Yunnan. Later, the Naxi people moved in in large numbers, they lived with the Tibetans in the Tibetan areas of Yunnan. Some Naxi people were assimilated into Tibetans, some were lived with local Tibetans, and some Naxi people retained their own living patterns, thus forming the Naxi village in the Tibetan area that witnesses the great integration of ethnic groups.

\section{The Great Unification Period of the Yuan Dynasty}

After the 13th century, Chinese entered a period of great unity, which was very prominent in the relationship between the Tibetan areas and the mainland. At the beginning of the 13th century, the Mongolian army defeated the Jin Dynasty and Xia Dynasty. The Tibetans in Gansu and Qinghai complied with the times and chose to submit. During the Wokuotai Khan period, Tibet's Saban chaidenzan led his two nephews: Ba Enba and Qiana Duoji, to meet Kuoduan, the ruler of Gansu and Qinghai designated by Mongolia and expressed his desire to submit, which promoted the unity of Mongolia and Tibet. Kublai Khan resolved to realize the great reunification of all ethnic groups in China since his accession to the throne to the founding of the Yuan Dynasty in 1271. He inherited the practice of Genghis that respects and utilizes the special influence of Tibetan religious beliefs on political forces. He appointed Ba Enba as National Master, and made him responsible for administrative affairs in all religious and Tibetan areas, and under which there were three Pacification Commissioner's Commandery, including Tubo Pacification Commissioner's Commandery charging parts of now Changbu, Tibet, the south of the two Tibetan autonomous prefectures of Aba and Ganzi, Deqin in the Tibetan areas of Yunnan, Shangsijing of Zhongdian, and the northwestern part of Weixi.

In the governance of the frontier and ethnic areas, the Yuan Dynasty government vigorously used the local aristocracy or people in the upper level based on the idea of "satisfying its people according to its folk custom", and vigorously promoted the system of relying on local upperlevel people to perform local political power management, thus forming the chieftain system since the Ming and Qing Dynasties. For Tibetan areas, they utilized both monks and folks, both military and civilians to promote their own rule. The Tibetan area of Yunnan was part of the Kang District at this time. Until the early Qing Dynasty, the Tibetan local authorities also regarded it as a part of the Kang District. The local officials were served by the local upper level. At that time, there were two "Diwa" in Zhongdian. One was assigned to the Nongsi family, that is, the Song family, and the other was appointed to the Lazan family, which was later called the Qi family. Both of them are folks, not monks. Therefore, the Tibetans in Zhongdian said that the earliest official of them was Qi and Song family. Their titles have been passed down from generation to generation, and their descendants are Qi Shichang and Song Guozhu, who were still in the post of chieftain before the liberation. At that time, Zhongdian County was actually divided into three Zongka. The area under the jurisdiction of Duke Zongka was centered on the central town of Zhongdian County today; the area under the jurisdiction of Gezi Zongka was generally the Xiaozhongdian District of today, and the boundary between the two Zongka is the river in the middle section of today's Jingkou. The E'ri Zongka, centered on the Nairi of the Liberation Township today, was generally bounded by Raosimogang and Duke Zongka.

The rulers of the Yuan Dynasty became the master of Tibetan Tubo and placed Tibet under the direct jurisdiction of the central dynasty, thus strengthening the ties between the Tibetan areas of Yunnan and the vast Tibetan areas and the mainland. Due to the complicated political, economic and ethnic relations in Yunnan, and the locality separatist powers of chieftains, the economic development is very unbalanced. The Ming government promoted the chieftain system, and arranged either local official or expatriates, or both, based on the different conditions in different places to strengthen their rule over the Yunnan frontier. The chieftain system promoted by the Ming Dynasty was a system using the local ruling group to rule the localities under the combination of the actual situation inherited from the history of the border based on the experience of the feudal rulers in governing the frontier ethnic groups in the past. It can also be called the system of "harmony co-existence after subjection", which is, in essence, the embodiment of the policy of "controlling foreigners by foreigners". [7] Such policy is very typical in the Tibetan areas of Yunnan, and has great influence on the development of ethnic relations in Yunnan Tibetan area during the Ming and Qing Dynasties and the Republic period (1912-1937).

During this period, in the migration and intermingling of multi-ethnic groups, the idea of Chinese unity was conceived and promoted, and the Tibetan areas in Yunnan gradually formed the idea of obedience and integration. With the formation of Chinese unity, the rulers in the Tibetan areas of Yunnan have basically reached a unified consensus, which not only makes the Tibetan areas in Yunnan form a deep unification complex under the long-term political confrontation and conflict, but also inspires the way of thinking and the scale of governance in the Tibetan areas in Yunnan. Such natural political value orientation of Chinese unity supports the symbiosis of the pluralistic unity of the Chinese nation and promotes the construction of a unified multi-ethnic country. 


\section{The Period When Gushi Khan of the Heshuote Tribe of Mongolia Defeated Chieftain $\mathrm{Mu}$}

During the Kangxi period in the early Qing Dynasty, the Qing Dynasty sent troops to Tibet to realize great unification. A large number of Man and Han officers stationed in Zhongdian and other places, which had a tremendous impact on the ethnic integration of Yunnan Tibetan area. The social history of Yunnan Tibetan area after the Qing Dynasty can be divided into two periods. In the early stage, the local separatist forces were gradually removed; The Yunnan Tibetan area were subject to the jurisdiction of Yunnan, and finish the bureaucratization of native officers, which was equivalent to the mid-17th century to the first half of the 18th century; the later period was the slow development and turbulent period of the Yunnan Tibetan community, equivalent to the mid-18th century to the early 20th century.

From the mid-17th century to the first half of the 18th century, the changes and development of the Tibetan community in Yunnan were inseparable from the changes in the political and religious forces surrounding it, especially in Tibet. In the late Ming and early Qing dynasties, Gushi Khan of the Heshuote tribe of Mongolia defeated Chieftain Mu, and occupied Jiantang (now Jiantang Town, Shangri-La), designated Duke zong. In the 10th year of Shunzhi, the Qing Dynasty officially enshrined the Fifth Dalai Lama, which showed that the Qing government had already determined to support the ruling policy of Gelu clique headed by the Dalai Lama in Tibet, and the administrative power of Tibet was granted to Gushi Khan. At this time, the Fifth Dalai Lama dispatched personnel to manage the Tibetan areas in Yunnan, and implemented the "Diba" system of Tibet ("Diba" is pronounced "diwa" in the Tibetan dialect of Diqing.). The entire Tibetan area of Yunnan was under the dual leadership of Tibet's Dalai Lama and Qinghai, Mongolia, which strengthened the rule over the Tibetan areas of Yunnan. "Shuka" organization was established to manage all regions from political, economic, and social aspects. There is a strict hierarchy within the "Shuka" organization. It has distinct class content, that is, the local official must be obeyed both in organization and thought, so do the monastery. "Shuka" organization itself is derived from the Tibetan serf society, which is easy to be accepted by the people of Tibetan areas and is conducive to national stability, promoting the exchange and integration of all ethnic groups. During this period, there emerged large number of Mongolian and Tibetan soldiers, merchants and nomadic people in Tibetan area of Yunnan and other areas, and Tibetan, Naxi, Mongolian, Han, Manchu and other multi-ethnic activities, which made the ethnic relationship in Zhongdian and other places particularly complicated, leading to another confrontation after the entering of Naxi people in the Ming Dynasty. After many confrontations, with the collapse of the Ming Dynasty, Chieftain $\mathrm{Mu}$ in Lijiang has lost the backbone of its rule. The Qing government established its rule over the Tibet region, eliminated the separationist rule and separatist forces of local chieftains, and achieved the policy of great unification. The Tibetan areas in Yunnan are directly managed by the central government, which further demonstrates the characteristics of mixed-living, integration and culture exchange of multi-ethnic groups. The Tibetan history and culture in Yunnan Tibetan area have distinct local features and become a highlight of local cultural development.

Because of the long-term political confrontation and conflict, the distinctive ethnic diversity features were gradually formed amidst the inter-embedded pattern of ethnicity. Of course, there exist huge gaps in the degree of civilization and development of ethnic groups in Tibetan areas of Yunnan, especially the development gap between the main ethnic groups and ethnic minorities because of the influence of natural conditions, history and culture, space and ability of national development and other factors. Before the democratic reform, there was still an obvious dual structure of such pattern, which not only has a huge impact on the deep construction of the multi-ethnic complementary integration in Yunnan Tibetan area, but also affects the communication and mutual benefit between different ethnic groups. However, the interwoven and interdependent interembedded distribution pattern of ethnic groups in the Tibetan areas of Yunnan, and the integration and promotion of Tibetan, Naxi, Li-su, Bai, Yi, Hui and other multi-ethnic groups, has indeed achieved inter-embedded promotion and co-regional identity of multi-ethnic groups.

\section{The Formation ANd DeVElopment OF MUlTi- ETHNIC SYMBIOSIS PATTERNS IN TIBETAN AREAS OF YUNNAN}

\section{A. Multi-ethnic Mixed Living with Difference in Ethnic Groups and Sympatric Dependency}

The long-term political disputes and confrontation objectively strengthened the national consciousness. When different national subjects ruled Yunnan Tibetan area, the main ethnic groups were all dominant nationalities, and other ethnic groups became second-class nationalities, reconstructing ethnic boundaries. The superiority of the main ethnic group during this period will strengthen the "instrumental" national identity to a certain extent. On the one hand, the natural existence of difference in ethnic groups will make the main ethnic group in the ruling class construct different social classes in a social and political way, strengthen the national identity, and in addition, the realities of sympatric dependency, such as same living habits, same living geography, cultural and religious backgrounds. Neighbors need to help each other at critical moments such as weddings and funerals, agricultural production, housing construction, etc., so as to avoid being isolated by other villagers and neighbors. Such traditional way of production and life goes beyond the simple multi-ethnic identification, and further weakness the identity of various ethnic groups.

Frequent exchanges between ethnic groups can lead to a weakening of the national identity. At the same time, it is of utmost importance that the living pattern of multi-ethnic mixed living in Yunnan Tibetan area determines the blending of life, production, culture and religion among ethnic minorities. Due to the long-term mixed living of multi-ethnic groups, ethnic groups have frequent exchanges in agricultural activities, economic transactions, weddings and funerals, etc., which have strengthened mutual learning 
and exchanges among various ethnic groups in Yunnan Tibetan area, and promoted the continuous development of ethnic groups in Tibetan areas in Yunnan, thus promoting the harmonious coexistence of various ethnic groups in Yunnan Tibetan area, and increasing the factors of intermingling and symbiosis in Tibetan areas of Yunnan. In addition, the ethnic groups in Yunnan Tibetan area have gradually gained certain convergence in terms of clothing, language, and forms of living. The inter-ethnic communication between different ethnic groups is increasing, and the structural differences among different ethnic groups are not big. It can be seen from these phenomena that the residents' living in the multiethnic mixed areas in the Tibetan areas of Yunnan have gradually merged, forming a trend of great integration of the nation.

At the same time, "cultural and ethnic identity" such as production mode, costumes, language, customs and habits serves as a factor to distinguish ethnic differences and an important basis for dividing ethnic boundaries. The multiethnic mixed living in the Tibetan areas of Yunnan makes the ethnic boundaries of the various ethnic groups less obvious. From the perspective of the multi-ethnic symbiosis in the Tibetan areas of Yunnan, the long-term mixed living of various ethnic groups has gradually deepened the contact and communication between different ethnic groups, thus forming a more convenient "inter-ethnic common language"; Cultures of different ethnic groups have been integrated, the cultural differences have gradually diminished, and the cultural boundaries have gradually become blurred. Because of the commonalities of the religious beliefs of various ethnic groups, the religious beliefs among the various ethnic groups interact and closely contacted, thus reflecting the characteristics of multi-ethnic harmony and coexistence. In the Tibetan areas of Yunnan, the mutual influence and mutual infiltration of multi-ethnic mixed living has made the customs and habits of different styles gradually converge in reorganization and integration, forming a new system of mutual recognition of all ethnic groups. With the frequent exchanges between various ethnic groups in Yunnan Tibetan area, the characteristics of their "cultural identity" have not been particularly obvious, and the trend of fuzzification has replaced the ethnic boundaries.

\section{B. Multi-ethnic Symbiosis Pattern of the Integration of Politics and Religion}

The ancestors of various ethnic groups in Yunnan Tibetan area were in a state of nomadic and hunting life in the early days. Before the Tang Dynasty, the national rule of feudal serfs had not directly reached the Tibetan areas of Yunnan. After the seventh century AD, the forces of Tang, Nanzhao, and Tubo had fight here, but they had not formed a long-term stable rule, nor could they change the social form of slavery in Yunnan Tibetan area. After the Yuan Dynasty, the central government promoted the policy of "the people are not listed and the soil is not ruled" in the Tibetan areas of Yunnan. Because of the introduction of Tibetan Buddhism and its deepening influence, the temple became the center of the dominant forces in Yunnan. Foreign ruling classes, such as the Chieftain $\mathrm{Mu}$ of the Ming Dynasty, used the power of religious monks and the power of the chieftain to maintain their rule, and gradually evolved into the ruling system of "the integration of politics and religion" in Yunnan Tibetan area. During the Yongzheng period of Qing Dynasty (AD 1723-1735), "bureaucratization of native officers" was implemented. Although many expatriates were dispatched, these officials in the Tibetan areas of Yunnan can only been seen but not heard. In fact, they have to rely on the power of monastery and chieftain. In most cases, the "the integration of politics and religion" ruling power formed by the upper monks, local chieftains and expatriates in the Tibetan areas of Yunnan has become increasingly apparent. In the Qing Dynasty, a complete and deeply entrenched "political and religious unity" system was formed. Under this system, Yunnan Tibetan area has always been in the feudal serf system and slavery society. The feudal landlord system was also formed in the later period, but until the establishment of the People's Republic of China, the remnants of the feudal serf system and slavery were not completely eliminated.

The highest ruling organization under the system of "political and religious unity" in the Tibetan area of Yunnan is the "Chuiyun Conference", which is the organization of the joint dictatorship and management of the aristocrats and monks. It is the local highest administrative conference institution in the Tibetan area of Yunnan at that time. It enjoys decision-making power of political, economic, cultural, religious, military and other major events in Yunnan Tibetan area and manages the political, economic and military affairs of the entire Tibetan region. The most common political and religious resolutions of the "Chuiyun Conference" are "apologetics" and "following the old practice of Tubo." Under the "Chuiyun Conference", there are two complete ruling systems. One is the temples in the Tibetan area of Yunnan manage monks system through "Zhacang"(A school specializing in the study of Buddhism), "Kangcan"(A group of monks) and "Mican", and implement feudal serfdom throughout the Tibetan area of Yunnan. The other is the chieftain control system, that's, to rule Yunnan Tibetan area through camp officials, Qianzong (junior military officers in the Qing Dynasty), and Bazong (junior military officers of Ming and Qing Dynasties), under which there is grassroots authorities: "Shuka", generally composed by cooks, the elder, and representatives of "Mican". A "Shuka" generally covers one or several natural villages.

The ethnic minorities living at the bottom of the Tibetan area in Yunnan at that time were partly serfs and some were slaves. Some of the serfs are ethnic minority officials and civilians who are subject to public rent. They are under the jurisdiction of chieftain. The public rent they undertake is called "Imperial Rations"; the other part is the minority religious people in the Tibetan areas of Yunnan, and they are responsible for the land rent around the temple and various crvees, and they collect all kinds of apportionment except tax. The serfs of the local ethnic minorities can only set up an independent house with the permission of the chieftain and the temple. Only after the establishment of the house can they have access to share the land, and become "people with permanent residence". Minorities without independent house have no land and no other rights. The land rented by minority 
serfs in the Tibetan areas of Yunnan is generally referred to as lotment. Although the minority serfs who rent the land have certain personal freedom, in addition to the land rent, they must do free labor, and bear all kinds of exorbitant taxes. Such unpaid labor is known as "Wulachai" (corvée labour formerly imposed on Xizang serfs.). The serfs of the ethnic minorities cannot leave lotment at will, and are in fact bound to the territory of the serf-owner forever. Before the founding of the People's Republic of China, feudal serf owners who accounted for about $1 \%$ of the total population of Yunnan's Tibetan areas occupied most land in Yunnan's Tibetan areas; $87 \%$ of the serfs in Yunnan's Tibetan areas had only a small amount of land, and about $30 \%$ owned no land. [7] In addition to serfs, slaves who make up about $5.4 \%$ of the total population of Yunnan Tibetan area have no personal freedom. The slaves in Tibetan areas in Yunnan are either bankrupt serfs, or prisoners of enemy, or bought from outside. They are slaves for generations.

The temple is the largest landowner in the Tibetan area. Songzanlin temple is the largest landowner in Tibetan areas of Yunnan at that time. In addition to two large estates, it covers eight "Shuka" and covers an area of 200,000 mu. Besides, it also occupies 3,377 mu of good farmland in Jinsha River Valley, accounting for $41.8 \%$ of the local leased land. [7] The land of temple consists of land donated by tenants who receive the lotment, land for debt repayment by the tenants, and land purchased by the temple. There are two main ways in which temples operate land. One is the feudal lord system, that is, to collect physical rent or labor rent from the serf who has the right to receive lotment and dispatch "Wulachai", and the other is tenancy management that includes flexible rent management (When landlords lease their land, they do not stipulate the land rent but set a certain rate of rent or, depending on the growth of the crops, decide on the number to be divided by both parties.) and fixed land rent management. The former means the crops should be equally divided by temple and the peasants, while the latter is fixed land rent collected due to the difference in land in Yunnan Tibetan area. Through the management of the land, the temple can gain a good fortune. In addition to voluntary donations, the religious income of the temple also includes apportioned income, that is, fixed apportionment and temporary apportionment of serfs from time to time. In addition to the above, the temple also increases the income by issuing usury loans. The usury loans issued by the temples generally have two categories: silver money debts and physical debts. Even the upper-level aristocratic families may become debtors.

During this period, the salaries of the local officials were highland barley collected in accordance with the grade in the form of land rent or others. After bureaucratization of native officers, the Qing Dynasty established a system of control in Zhongdian and Weixi, and collected tax items such as silver, highland barley, summer tax, and autumn grain by dispatching expatriates. During the Xuantong period of Qing Dynasty, a working organization was set up in today's Adunzi of Deqin to collect the salt tax on salt wells in Tibet. The taxation of Weixi County is relatively heavy. After the first year of Emperor Qianlong (AD 1736), various taxes that were submitted by the Yunnan Tibetan area each year totaled 1093 liang (50g) silver. [7] Apart from temple and chieftain, the common people also increased the burden of expatriates in government office in feudal China.

The long-standing political system of the combination of politics and religion in the Tibetan areas of Yunnan, especially the maintenance of the political forces of the temple, is on the one hand based on the influence of religious traditions such as Tibetan Buddhism, and on the other hand based on certain coercive forces, such as the armed force in temple. The armed force in temple is the capital and support of the upper monks in the temple to participate in various political struggles. There are prisons in temples and chieftain mansion. Generally, there are related customary laws and local village statutes. The cases are usually judged by chieftain or temple. The major cases involving the ruling class in the jurisdiction area will be submitted to the "Chuiyun Conference" for final decision.

In the process of governance of the integration of politics and religion in Tibetan areas of Yunnan, the symbiotic pattern of multi-ethnicity has been further consolidated and strengthened. Tibetans, Naxi, Li-Su and other ethnic minorities have achieved mutual integration in the confrontation and struggle of various political forces. Many religions have gradually coexisted, and national culture has gradually been preserved. The interwoven and interdependent symbiotic pattern is developing rapidly.

\section{CONCLUSION}

The historical formation of multi-ethnic integration symbiosis in the Tibetan areas of Yunnan has played a major foundation role in the prosperity and stability of the frontiers and the progress of multi-ethnic unity. During the long-term historical evolution, the identification of interdependence towards the same region of various ethnic groups in Tibetan areas of Yunnan has formed. In the context of ethnic differences, the pattern of multi-ethnic symbiosis has been further consolidated and strengthened, realizing long-term prosperity, stability, development and progress. Tibetans, Naxi, Li-Su and other ethnic minorities have achieved mutual integration in the confrontation and struggle of various political forces. Many religions have gradually coexisted, and national culture has gradually been preserved. The interwoven and interdependent symbiotic pattern is developing rapidly.

\section{REFERENCES}

[1] Statistics Bureau of Diqing Tibetan Autonomous Prefecture Statistical Communiqué on National Economic and Social Development in 2015 [J], Diqing Daily, 2016(4): 8. (in Chinese)

[2] Li Zhinong. Exploration on the Religious Relationship in Diqing Tibetan Areas in Yunnan $[\mathrm{J}]$, Research on Southwest Frontier Nationalities, 2007(8): 35-41. (in Chinese)

[3] Yang Xuli. Diqing History [M], The Nationalities Publishing House of Yunnan, 2007(5): 102-103. (in Chinese)

[4] Feng Zhi. Research on Tibetan Studies in Yunnan: The Relationship between Politics and Religion in Tibet and the Tibetan System in the 
Qing Dynasty [M], The Nationalities Publishing House of Yunnan, 2007(3): 17. (in Chinese)

[5] Mu Jihong. Exploring the Culture of Yunnan, Tibet and Sichaun [M], Yunnan University Press, 1992(7): 26. (in Chinese)

[6] Wang Hengjie. Social History of Diqing, Tibetan [M], China Tibetology Press, 1995, (8): 48. (in Chinese)

[7] Le Anwangdui. A Brief History of Contemporary Yunnan Ethnic Minorities: A Brief History of Contemporary Tibetans in Yunnan [M], The Nationalities Publishing House of Yunnan, 2009(9): 26-47. (in Chinese) 